\title{
Respiratory muscle strength in chronic stroke survivors and its relation with the 6 -minute walk test
}

\author{
Ana Lista Paz, Luz González Doniz, Serafín Ortigueira García, Jesús Luis Saleta \\ Canosa, Christian Moreno Couto
}

\begin{abstract}
Objectives. To compare respiratory muscle strength in stroke survivors (SS) with that in a control group (CG) of healthy people matched by age and sex, as well as to investigate any relation between respiratory muscle strength and the distance walked during the 6-minute walk test (6MWT).

Design. Cross-sectional study.

Setting. This study comprised patients from a private neurological rehabilitation center and a public association for patients with acquired brain injury.

Participants. Chronic SS with a diagnosis of hemiplegia/hemiparesis who were able to walk $(\mathrm{n}=30)$ and healthy individuals matched by sex and age $(\mathrm{n}=30)(\mathrm{N}=60)$.

Interventions. Not applicable.

Main Outcome Measures. Respiratory muscle strength was assessed using maximal expiratory pressure (MEP) and maximal inspiratory pressure (MIP) with a pressure transducer and a diver nozzle. The distance walked during the $6 \mathrm{MWT}$ was also registered for both groups. The motricity index and the Scale Impact of Stroke version 16.0 were also measured in SS.

Results. The Student $t$ test revealed significantly lower values of MIP and MEP in SS than in the CG (MEP, $95.93 \pm 43.12 \mathrm{cmH}_{2} \mathrm{O}$ in $\mathrm{SS}$ vs $158.43 \pm 41.6 \mathrm{cmH}_{2} \mathrm{O}$ in the $\mathrm{CG}$; $\mathrm{MIP}, 58.7 \pm 24.67 \mathrm{cmH}_{2} \mathrm{O}$ in $\mathrm{SS}$ vs $105.7 \pm 23.14 \mathrm{cmH}_{2} \mathrm{O}$ in the CG; $P<.001)$. Moreover, these findings were clinically relevant because both MIP and MEP in SS were $<60 \%$ of the predicted values. A positive correlation between the 6MWT and MIP was found using the Pearson coefficient $(r=.43 ; P<.018)$.

Conclusions. Respiratory muscle weakness should be considered in a holistic approach to stroke rehabilitation.
\end{abstract}

Keywords

Physical therapy modalities; Rehabilitation; Respiratory function tests; Respiratory muscles; Stroke

List of abbreviations

6MWT, 6-minute walk test; CG, control group; MEP, maximal expiratory pressure; MIP, maximal inspiratory pressure; SS, stroke survivors

Stroke is a major cause of disability in adults worldwide and is a key social health concern in Spain, where an estimated annual incidence is 252 per 100,000 people. ${ }^{1}$ and 2 Some respiratory problems have been identified immediately after the stroke during the acute and subacute period, such as sleep disorders, ${ }^{3}$ altered ventilatory patterns, ${ }^{4}$ and 5 and risk of aspiration. ${ }^{6}$ However, neurological rehabilitation approaches to chronic stroke survivors (SS) rarely take into account assessment and treatment of respiratory muscles. A literature review $^{7}$ carried out in 2011 showed that patients with chronic hemiplegia/hemiparesis after a stroke have limitations in their static and dynamic volumes and present respiratory muscle alterations and concluded that these responses should be considered during patient rehabilitation. Few studies ${ }^{8,9,10}$ and 11 published in the past 10 years have assessed respiratory muscle strength in patients with stroke, gauging maximal expiratory pressure (MEP) and maximal inspiratory pressure (MIP). A systematic review ${ }^{12}$ published in 2013 concluded that SS have respiratory muscle weakness, which could be correlated with a higher risk of chest infections in that population. A significant decrease in peak cough flow has also been observed in subacute ${ }^{13}$ and chronic ${ }^{14} \mathrm{SS}$ compared with a control group (CG) of healthy individuals, which has been related to a lack of respiratory muscle strength and coordination. ${ }^{15}$ Respiratory muscle strength has been poorly studied in chronic SS, and there are several differences in the methodology and protocols for its measurement. A decrease in MEP in 
patients with hemiplegia has been related to a lack of trunk control, ${ }^{16}$ whereas a lower value of MIP has been correlated with a decrease in gait speed. ${ }^{17}$

The primary aim of this study was to compare respiratory muscle strength in SS with that in a CG of healthy people matched by age and sex. The secondary aim was to investigate any relation between respiratory muscle strength and the distance walked during the 6-minute walk test (6MWT).

\section{Methods}

Study design

Data in this article are a subset of baseline data from a cross-sectional study of pulmonary function in patients with chronic hemiplegia/hemiparesis after a stroke as compared with a CG of healthy people matched by age and sex.

\section{Participants and setting}

The present study was conducted with patients from a private neurological rehabilitation center and a public association for patients with acquired brain injury, both located in Galicia, Spain. The recruitment was carried out from January 15, 2012 to October 15, 2014. Thirty-three patients with chronic hemiplegia/hemiparesis were recruited from a potential sample of $146 \mathrm{SS}$ and filtered using the following criteria in accordance with the principal study.

\section{Inclusion criteria}

We included individuals if they had a diagnosis of hemiplegia/hemiparesis and $\geq 6$ months have passed since the onset of the stroke; were aged $>18$ years; were receiving neurological rehabilitation treatment; were able to walk with or without assistance (assistive device, orthotic device, and/or physical aid from another person); were able to understand instructions for several tests; demonstrated a willingness to participate in the study.

\section{Exclusion criteria}

We excluded individuals who were current or ex-smokers for $\geq 1$ year; had a diagnosis of pulmonary disease; had a diagnosis of severe cardiovascular disease; had any other neurological impairment; had experienced thoracic or abdominal surgery in the previous 3 months; had severe facial paralysis; had severe kyphoscoliosis; and were receiving specific cardiovascular training or respiratory physiotherapy.

Thirty-three individuals in the CG were selected from the staff of the University of A Coruña and from the users of a local residential home for the elderly by using the same criteria described for SS. The individuals in the CG were matched by age and sex with SS because those variables can influence the results of maximal respiratory pressures. ${ }^{18}$

This project was approved by the Clinical Research Ethics Committee of Galicia. All individuals gave their written consent to participate.

\section{Procedure}

Participant assessments were carried out in the Faculty of Physiotherapy, University of A Coruña. A specially trained physiotherapist conducted all measurements in 2 sessions.

\section{First visit}

All participants underwent a standardized interview about their demographic and clinical characteristics and rehabilitation procedure details. Anthropometric and vital signs (heart rate, breath rate, oxygen saturation level, blood pressure) were also noted. Two 6MWTs were conducted in both groups in an indoor corridor (30m long, with each meter marked) that was seldom traveled, in line with the international statements. ${ }^{19}$ Before starting, clear indications for the test were given to the participants. The principal instruction was to "walk as fast as you can without running." Standardized verbal encouragement, described for the validated Spanish version, ${ }^{20}$ was given. The participants in the SS group 
were allowed to use their customary assistive/orthotic devices. When physical or supervision aid from another person was needed, we trained a companion (normally a relative) to walk with the participant without influencing his/her gait speed. Individuals had to rest for $\geq 30$ minutes before starting the second test. Distance (in meters) and speed gait (in meters per second) were calculated.

To characterize the sample, additional tests were performed. Motor function in patients with stroke was assessed through the motricity index ${ }^{21}$ using the protocol described by Collin et al. ${ }^{22}$ We used the Scale Impact of Stroke version $16.0^{23}$ to assess the consequences of the stroke. The Scale Impact of Stroke version 16.0 has been validated for the Spanish population. ${ }^{24}$

\section{Second visit}

MEP and MIP were measured, in line with the international statements, ${ }^{25}$ using a pressure transducer (model 511-8D0-MU1 ${ }^{\text {a }}$ ) with an operating interval of $\pm 300 \mathrm{cmH}_{2} \mathrm{O}$ and a precision of $3 \%$ connected to a spirometer Datospir 120C. ${ }^{\text {a }}$ Equipment calibration was performed according to the international standards. $^{25}$ All measurements were taken in a sitting position with a rubber diver nozzle (scuba mouthpiece) and a nose clip, with participants asked to sustain pressures for 3 to 5 seconds. ${ }^{26}$ The pressure transducer calculated the maximal peak pressure generated after the first second (the first second is discounted because of inertia). Participants were asked to hold their cheeks rigid with their hands to avoid leaks and to minimize buccinator muscle contributions during the maneuvers. In the case of a subject with moderate facial paralysis, as described in the literature ${ }^{27}$ an assistant physiotherapist held the participant's lips around the mouthpiece to ensure a perfect seal between lips and the nozzle. When an SS was unable to adequately move an upper limb, an assistant would manually support the patient's cheeks. We performed a maximum of 10 repetitions to measure MEP and MIP values, with a minimum of 6 acceptable maneuvers, 3 of them with variability $<5 \%$ (repeatability criteria). ${ }^{26}$ We chose the highest reading of the 3 reproducible maneuvers. The participants had to rest for 1 minute between each repetition of MEP and 5 minutes before starting the MIP repetitions. The Morales' predictive equations, ${ }^{28}$ created from a healthy sample of Spanish people, were used in this study.

\section{Data analysis}

A descriptive analysis of registered variables was performed. Demographic quantitative variables are presented as mean $\pm \mathrm{SD}$, whereas qualitative variables are presented as absolute values. MIP and MEP were also expressed as percentages of predictive values. After testing for normal distribution of quantitative variables using the Kolmogorov-Smirnov test, the Student $t$ test was used to determine differences in maximal respiratory pressures between SS and the CG. Moreover, Student $t$ tests were performed to determine whether there were any differences between groups with regard to age, weight, height, and reference values of MEP and MIP. The significant level was set at $P<.05$.

To test correlations between MEP and MIP and the distance walked during the 6MWT, the Pearson coefficient was used with the accepted significance level of $P<.05$. Dancey and Reidy's categorization ${ }^{29}$ was subsequently used to interpret the strength of the correlation.

All statistical analyses were performed using SPSS version 22.0. ${ }^{\mathrm{b}}$

\section{Results}

Sixty-six participants were recruited for this study (33 per group). After the first interview, 2 individuals in each group were withdrawn because they presented contraindications for the performance of maximal respiratory pressures (problems in the eardrum and recent ocular surgery). One subject in each group was not included in the final analysis because they were not able to adequately achieve MEP and MIP values after 10 repetitions of each. We present in this article a sample of 60 participants, 22 (73\%) men and $8(27 \%)$ women per group. Table 1 shows that there were no significant differences between groups with regard to age, weight, height, and body mass index. Among SS, 11 participants had an affected right side whereas 19 had an affected left side. The average length of time since the stroke was 45.55 \pm 66.08 months (range, 6-324mo). Most SS were inactive (unemployed, retired, or receiving a disability pension), and only 2 were employed. All SS were recipients of physiotherapy treatment, with an average treatment of $2.47 \pm 1.27 \mathrm{~h} / \mathrm{wk}$. Moreover, 19 participants were receiving occupational therapy in the same centers, with an average treatment of $1.58 \pm 1.3 \mathrm{~h} / \mathrm{wk}$. Further details and outcomes achieved in the 6MWT, the motricity index, and the Scale Impact of Stroke version 16.0 are given in table 1. 
Table 1. Characteristics of the individuals $(n=60)$

\begin{tabular}{|c|c|c|c|c|c|c|}
\hline \multirow{2}{*}{ Variable } & \multicolumn{2}{|c|}{$\mathrm{SS}(\mathrm{n}=30)$} & \multicolumn{2}{|c|}{$\mathrm{CG}(\mathrm{n}=30)$} & \multicolumn{2}{|c|}{$\begin{array}{c}\text { Differences Between } \\
\text { SS and CG }\end{array}$} \\
\hline & Mean \pm SD & $\mathrm{n}$ & Mean \pm SD & $\mathrm{n}$ & Diff. Means & $P$ \\
\hline Sex: male/female & NA & $22 / 8$ & NA & $22 / 8$ & NA & NA \\
\hline Age (y) & $55.60 \pm 15.84$ & NA & $55.33 \pm 14.61$ & NA & .27 & .95 \\
\hline Height $(\mathrm{cm})$ & $166.27 \pm 9.5$ & NA & $169.07 \pm 9.07$ & NA & -2.8 & .25 \\
\hline Weight $(\mathrm{kg})$ & $77.79 \pm 15.1$ & NA & $77.82 \pm 13.95$ & NA & -.027 & .99 \\
\hline BMI (kg/m2) & $28.02 \pm 4.74$ & NA & $27.15 \pm 3.97$ & NA & .87 & .44 \\
\hline Type of stroke: I/H & NA & $23 / 7$ & NA & NA & NA & NA \\
\hline \multicolumn{7}{|l|}{ Lesion location } \\
\hline $\mathrm{MCA} / \mathrm{ACA} / \mathrm{MCA}+\mathrm{ACA}$ & NA & $21 / 2 / 1$ & NA & NA & NA & NA \\
\hline Lobar/BG/BS & NA & $3 / 2 / 1$ & NA & NA & NA & NA \\
\hline Affected side: right/left & NA & $11 / 19$ & NA & NA & NA & NA \\
\hline Months since the stroke & $45.55 \pm 66.08$ & NA & NA & NA & NA & NA \\
\hline Distance walked during the 6MWT (m) & $270.67 \pm 129.18$ & NA & $702.93 \pm 88.28$ & NA & -432.27 & $<.001 *$ \\
\hline Speed 6MWT (m/s) & $0.75 \pm .36$ & NA & $1.95 \pm 0.25$ & NA & -1.2 & $<.001 *$ \\
\hline LL_MI & $69.4 \pm 20.86$ & NA & NA & NA & NA & NA \\
\hline UL_MI & $57.77 \pm 33.18$ & NA & NA & NA & NA & NA \\
\hline Global_MI & $64.55 \pm 22.89$ & NA & NA & NA & NA & NA \\
\hline SIS-16 & $66.73 \pm 8.35$ & NA & NA & NA & NA & NA \\
\hline
\end{tabular}

Abbreviations: ACA, anterior cerebral artery; BG, basal ganglia; BMI, body mass index; BS, brain stem; Diff. Means, difference between means of both groups; I/H, ischemic/hemorrhagic; LL, lower limb; MCA, middle cerebral artery; MI, motricity index; NA, not applicable; SIS-16.0, Scale Impact of Stroke version 16.0; Speed 6MWT, speed gait during the 6MWT; UL, upper limb.

* Statistical significance.

Even though none of them were active smokers, $18 \mathrm{SS}$ and 14 people in the CG were ex-smokers. The average length of time since stopping smoking was 13.29 \pm 11.92 years in SS and 20.5 \pm 10.55 years in the CG.

Twelve SS needed an assistive device to walk (straight cane, $n=6$; crutch, n=4; a tripod, $n=2$ ). Five patients used a foot Dictus ${ }^{\mathrm{c}}$ drop brace. Seven participants needed some kind of physical assistance to walk ( 2 required physical support, whereas 5 needed only supervision).

Table 2 shows the results of MEP and MIP for both groups as a mean of absolute value and as a percentage of the predictive value, calculated using the Morales' equations. ${ }^{28}$ 
Table 2. Maximal respiratory pressures in the SS and CG $(n=60)$

\begin{tabular}{lccccc}
\hline Variable & $\mathrm{SS}(\mathrm{n}=30)$ & $\mathrm{CG}(\mathrm{n}=30)$ & Diff. Means & $P$ & $95 \% \mathrm{CI}$ \\
\hline & & & & & \\
$\mathrm{MEP}\left(\mathrm{cmH}_{2} \mathrm{O}\right)$ & $95.93 \pm 43.12$ & $158.43 \pm 41.60$ & -62.5 & $<.001^{*}$ & 40.60 to 84.39 \\
r.v. $\mathrm{MEP}\left(\mathrm{cmH}_{2} \mathrm{O}\right)^{\dagger}$ & $174.24 \pm 31.42$ & $173.07 \pm 32.43$ & 1.17 & .889 & -17.96 to 15.62 \\
Obs MEP/r.v. MEP $(\%)$ & $51.56 \pm 20.83$ & $95.62 \pm 19.06$ & -41.35 & $<.001^{*}$ & 33.56 to 54.56 \\
$\mathrm{MIP}\left(\mathrm{cmH}_{2} \mathrm{O}\right)$ & $58.7 \pm 24.67$ & $105.7 \pm 23.14$ & -47 & $<.001^{*}$ & 34.64 to 59.36 \\
r.v. $\mathrm{MIP}\left(\mathrm{cmH}_{2} \mathrm{O}\right)^{\dagger}$ & $116.38 \pm 20.63$ & $115.28 \pm 18.89$ & 1.1 & .833 & -11.51 to 9.3 \\
Obs MEP/r.v. MIP $(\%)$ & $51.41 \pm 20.85$ & $93.1 \pm 19.69$ & -39.91 & $<.001^{*}$ & 30.94 to 52.46 \\
\end{tabular}

NOTE. Values are mean \pm SD

Abbreviations: CI, confidence interval; Diff. Means, difference between means of both groups; Obs, observed value; r.v., reference value.

* Statistical significance.

$\uparrow$ Reference values were calculated using Morales' predictive equations.

SS had significantly lower values of both MEP and MIP than did individuals in the CG (MEP, $95.93 \pm 43.12 \mathrm{cmH}_{2} \mathrm{O}$ in $\mathrm{SS}$ vs $158.43 \pm 41.6 \mathrm{cmH}_{2} \mathrm{O}$ in the CG; MIP, $58.7 \pm 24.67 \mathrm{cmH}_{2} \mathrm{O}$ in $\mathrm{SS}$ vs $105.7 \pm 23.14 \mathrm{cmH}_{2} \mathrm{O}$ in the CG; $\left.P<.001\right)$. Furthermore, in SS, MEP and MIP were $<60 \%$ of the predicted values $(51.56 \pm 20.83$ and $51.41 \pm 20.85$, respectively), which is considered muscle weakness in the literature. 18 and 30

SS walked a mean distance of $270.67 \pm 129.18$ m during the 6MWT. A moderate positive correlation $(r=.43 ; P<.018$ ) between the 6MWT and MIP was found using the Pearson coefficient ( table 3 ).

Table 3. Correlation between the 6MWT and MEP/MIP in SS

\begin{tabular}{lccc}
\hline Variable & Pearson Coefficient Correlation Strength* & $P$ (2-tailed) \\
\hline & & & \\
MIP & .430 & Moderate & $.018^{\dagger}$ \\
MEP & .245 & NC & .191 \\
\end{tabular}

Abbreviation: NC, no correlation.

* Dancey and Reidy's categorization was used to interpret the strength of the correlation.

$\dagger$ Statistical significance.

\section{Discussion}

The results of this study indicate a significant reduction in respiratory muscle strength in chronic SS as compared with a CG of healthy people measured by maximal static respiratory pressures. Outcomes also demonstrate a moderate positive relation between inspiratory muscle strength and the distance walked during the 6MWT.

In general, our findings are consistent with the literature. ${ }^{8,12,16 \text { and } 31}$ However, it is difficult to compare the results from the present study with other research findings because patients with stroke are a highly heterogeneous population. There are few studies conducted within 6 months of the stroke. Moreover, there are important differences in the methodology and measurement procedures.

One of the main differences between our study and other studies was related to the assessment procedure, in particular the equipment and performance of MEP and MIP. Studies ${ }^{8,17}$ and 32 commonly use a portable manovacuometer requiring participants to sustain maximal pressures for $\geq 1$ second. Instead of following the international standards, ${ }^{25}$ we used an electronic pressure transducer requiring the individuals to sustain the effort for $\geq 3$ seconds to achieve a plateau on the curves of MEP and MIP. 
Several of the consulted studies, 16 and 17 have registered 3 acceptable maneuvers, 2 of them reproducible with a maximum of $10 \%$ of variation. However, we aimed for 6 acceptable maneuvers (without leaks and with a plateau on the curves), 3 of them reproducible with a maximum of $5 \%$ of variation. We followed this procedure ${ }^{26}$ to achieve more accuracy and to minimize the learning effect because this can influence the results of MEP and MIP..$^{33}$ and 34

Most of the reviewed articles ${ }^{8,16,17 \text { and } 31}$ have not considered smokers or ex-smokers within the exclusion criteria. In our study, we excluded smokers and we accepted only ex-smokers (smoking for $\geq 1 \mathrm{y}$ ), because it has been observed that current smokers have $15 \%$ lower MIP than do non- or exsmokers. 35

In 1983, Fugl-Meyer et al $^{31}$ demonstrated a lack of respiratory muscle strength in 54 SS compared with a CG of 12 healthy people. They found a decrease in MIP and MEP in the CG, with a difference between MIP and MEP being 20\% of the predictive values. However, we found similar percentages in predictive values of MIP and MEP in SS (51\%). The results of both studies are not comparable because a different protocol was used for measurements and the time period after the stroke was not specified. FuglMeyer assessed MIP and MEP in the supine position and compared these outcomes with predictive seated normal values. MIP and MEP are significantly higher in the sitting position than in the supine position. ${ }^{36}$

In 2005, Teixeira-Salmela et $\mathrm{al}^{8}$ assessed respiratory muscle strength in 16 chronic SS compared with a CG of healthy people. They found a significant difference between both groups with regard to MEP and MIP. When they compared results with predictive values, they found an MEP of $90 \%$ and an MIP of $\sim 79 \%$. However, in our study, we achieved a clinical relevance of respiratory muscle weakness ${ }^{30}$ because both maximal respiratory pressures were $<60 \%$ of the predictive values of both MEP and MIP (51\%). These higher results of Teixeira-Salmela could be attributed to the differences in the aforementioned procedures as well as to the fact that participants with a restrictive ventilatory pattern were excluded. Some authors ${ }^{37}$ and 38 have pointed out that patients with chronic hemiplegia can present a restrictive respiratory pattern poststroke. Moreover, it has been reported that inspiratory muscle weakness could be responsible for vital capacity $<80 \%$ of the predictive value. ${ }^{18}$ The same research group published another $\operatorname{article}^{32}$ with a larger sample of chronic SS $(n=98)$, finding similar maximal respiratory pressures, expressed as absolute values (MIP, 73.5 $\pm 29.2 \mathrm{cmH}_{2} \mathrm{O}$; MEP, 81.6 $\pm 37.7 \mathrm{cmH}_{2} \mathrm{O}$ ). However, they did not show the percentage with respect to reference values using the same procedure as in 2005 .

In 2013, Pollock et al $^{12}$ conducted a systematic review with a meta-analysis, including 4 articles that compared MEP and MIP of SS with those of a CG of healthy people. They concluded that there was a significant decrease in respiratory muscle strength in this population as compared with healthy people. It is worth noting, however, that this systematic review pooled data from acute and chronic SS.

None of the cited original studies have provided the $95 \%$ confidence interval. However, the $95 \%$ confidence interval in this study shows that our results can be extrapolated to chronic SS with a high clinical relevance (see table 2).

The results of Jandt et $\mathrm{al}^{16}$ also confirm a decrease in MEP and MIP in patients with stroke, and Jandt found a positive significant correlation between MEP and trunk control, assessed with the Trunk Impairment Scale. Jandt observed a positive correlation between MIP and the Trunk Impairment Scale, which was near to statistical significance $(P=.054)$. These outcomes suggest a new research line to investigate whether inspiratory and expiratory muscle training in chronic SS could improve postural stabilization, which is especially important for functional tasks with these patients.

In 2014, Pinheiro et $\mathrm{al}^{17}$ demonstrated that chronic SS noncommunity ambulators (those who have a gait speed of $<0.8 \mathrm{~m} / \mathrm{s})^{39}$ have significantly lower MIP values than do community ambulators (those who have a gait speed of $\geq 0.8 \mathrm{~m} / \mathrm{s}$ ). ${ }^{39}$ This is in agreement with our findings of a positive correlation between MIP and the distance walked during the 6MWT. We wanted to highlight the clinical relevance of that point. Sutbeyaz et $\mathrm{al}^{10}$ demonstrated, through a randomized controlled trial, that inspiratory muscle training for the population with subacute stroke had significant improvements in exercise capacity and quality of life. This is in agreement with the reports by Kim et al, ${ }^{9}$ which showed that respiratory muscle training produces a significant increase in distance walked during the 6MWT. This is an important factor in enhancing the social participation of SS. In addition, it has been proved that feedback respiratory training increases chest expansion and pulmonary function parameters in patients with chronic stroke. ${ }^{40}$

Furthermore, a good level of muscle respiratory strength and coordination is needed to have an effective cough, allowing maintenance of a clear airway and protection against aspiration. ${ }^{41}$ In the previous cough phase, inspiratory muscle weakness does not permit the inhalation of sufficient air volume. Moreover, expiratory muscle weakness compromises the intrathoracic pressure needed to produce a powerful expiratory airflow. ${ }^{15}$ and 42 High incidence of pneumonia after stroke has been widely reported. ${ }^{43}$ It is higher in patients with swallowing difficulty (dysphagia) because of aspiration risk. ${ }^{44}$ and 45 With these factors in mind, it would be necessary to investigate whether a systematic assessment of respiratory muscle strength during the rehabilitation program of chronic SS contributes to the prevention 
of the risk of chest infections in that population. There is an emerging pilot study investigating whether respiratory muscle training in acute SS could improve cough flow and its effectiveness as well as whether there is a relation with the incidence of pneumonia. ${ }^{46}$ and 47

\section{Study limitations}

There are some limitations to consider in this study. Although we aimed for 6 acceptable maneuvers of MIP and MEP with a plateau on pressure curves, we could not achieve them in all participants. Figure 1 shows an example of the representative graphs of MEP and MIP for matched individuals from both SS and the CG. While measuring MIP and MEP, we observed that in some SS there is a visible increase in spasticity in their affected side, which may have restricted maximal efforts. This inconvenience has not been reported in the consulted literature. Future studies should have a larger sample, with a classification of different levels of motor impairment after a stroke, to correlate with MEP and MIP.

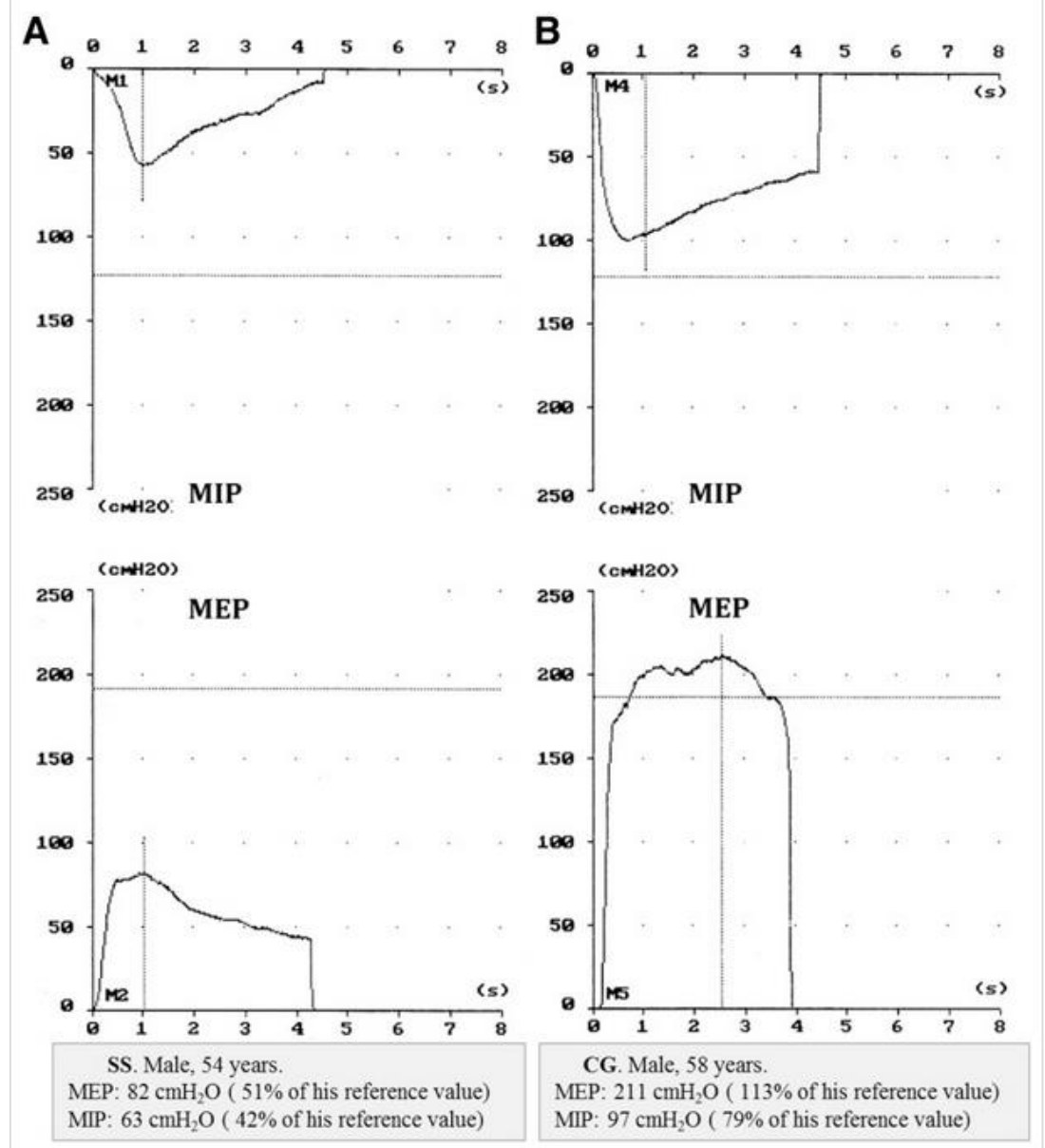

Fig 1. Example of representative graphs of MEP and MIP for matched individuals from both the SS group and the CG. (A) A 54-year-old male in the SS group. MEP, $82 \mathrm{cmH}_{2} \mathrm{O}(51 \%$ of his reference value); $\mathrm{MIP}, 63 \mathrm{cmH}_{2} \mathrm{O}$ (42\% of his reference value). (B) A 58-year-old male subject in the CG. MEP, $211 \mathrm{cmH}_{2} \mathrm{O}\left(113 \%\right.$ of his reference value); $\mathrm{MIP}, 97 \mathrm{cmH}_{2} \mathrm{O}(79 \%$ of his reference value). 


\title{
Conclusions
}

MIP and MEP decreased significantly in SS. Inspiratory and expiratory muscle weakness was observed as a clinical relevant finding. Moreover, a positive moderate correlation between MIP and the $6 \mathrm{MWT}$ has been demonstrated. A systematic assessment of respiratory muscle strength should be considered as a part of an integral approach to stroke rehabilitation.

\author{
Suppliers \\ a. Sibel Group. \\ b. IBM España S. A. \\ c. Erimed.
}

Disclosures: none

\section{References}

1. Brea A, Laclaustra M, Martorell E, Pedragosa A. [Epidemiology of cerebrovascular disease in Spain] [Spanish]. Clin Investig Arterioscler 2013;25:211-7.

2. Díaz Guzmán J, Egido J, Gabriel Sánchez R, Barberá Comes G, Fuentes Gimeno B, Fernández Pérez C. Stroke and transient ischemic attack incidence rate in Spain: the IBERICTUS study. Cerebrovasc Dis 2012;34:272-81.

3. Hermann D, Bassetti C. Sleep apnea and other sleep-wake disorders in stroke. Curr Treat Options Neurol 2003;5:241-9.

4. Bonnin Vilaplana M, Arboix A, Parra O, García Eroles L, Montserrat J, Massons J. Cheyne-Stokes respiration in patients with first-ever lacunar stroke. Sleep Disord 2012;2012:257890.

5. Rowat A, Wardlaw J, Dennis M. Abnormal breathing patterns in stroke: relationship with location of acute stroke lesion and prior cerebrovascular disease. J Neurol Neurosurg Psychiatr 2007;78:277-9.

6. Smith Hammond C, Goldstein L, Horner R, et al. Predicting aspiration in patients with ischemic stroke: comparison of clinical signs and aerodynamic measures of voluntary cough. Chest 2009;135:769-77.

7. Lista Paz A, González Doniz L. Pulmonary function in chronic hemiplegic/hemiparetic patients: a review of the literature [Spanish]. Rev Iberoam Fisioter Kinesiol 2011;14:38-45.

8. Teixeira-Salmela L, Parreira V, Britto R, et al. Respiratory pressures and thoracoabdominal motion in communitydwelling chronic stroke survivors. Arch Phys Med Rehabil 2005;86:1974-8.

9. Kim J, Park J, Yim J. Effects of respiratory muscle and endurance training using an individualized training device on the pulmonary function and exercise capacity in stroke patients. Med Sci Monit 2014; 20:2543-9.

10. Sutbeyaz S, Koseoglu F, Inan L, Coskun O. Respiratory muscle training improves cardiopulmonary function and exercise tolerance in subjects with subacute stroke: a randomized controlled trial. Clin Rehabil 2010;24:240-50.

11. Lanini B, Bianchi R, Romagnoli I, et al. Chest wall kinematics in patients with hemiplegia. Am J Respir Crit Care Med 2003;168:109-13.

12. Pollock R, Rafferty G, Moxham J, Kalra L. Respiratory muscle strength and training in stroke and neurology: a systematic review. Int J Stroke 2013;8:124-30.

13. Kimura Y, Takahashi M, Wada F, Hachisuka K. Differences in the peak cough flow among stroke patients with and without dysphagia. J UOEH 2013;35:9-16.

14. Yoon J, Park J, Lee D, Roh H. Comparisons of respiratory function and activities of daily living between spinal cord injury and stroke patients and normal elderly people. J Phys Ther Sci 2012;24:465-9.

15. Chang A. The physiology of cough. Paediatr Respir Rev 2006;7:2-8.

16. Jandt SR, Caballero RM, Junior LA, Dias AS. Correlation between trunk control, respiratory muscle strength and spirometry in patients with stroke: an observational study. Physiother Res Int 2011;16:218-24.

17. Pinheiro MB, Polese JC, Faria CD, et al. Inspiratory muscular weakness is most evident in chronic stroke survivors with lower walking speeds. Eur J Phys Rehabil Med 2014;50:301-7.

18. Evans J, Whitelaw W. The assessment of maximal respiratory mouth pressures in adults. Respir Care 2009;54:1348-59.

19. ATS Committee on Proficiency Standards for Clinical Pulmonary Function Laboratories. ATS statement: guidelines for the six-minute walk test. Am J Respir Crit Care Med 2002;166:111-7.

20. Vilaró J. Six-minute walk test [Spanish]. In: Scientific Committee of SEPAR. Manual procedures of SEPAR 4. Assessment evaluation of pulmonary function II. Barcelona: Sociedad Española de Neumología y Cirugía Torácica; 2004. p. 100-14.

21. Demeurisse G, Demol O, Robaye E. Motor evaluation in vascular hemiplegia. Eur Neurol 1980;19:382-9.

22. Collin C, Wade D. Assessing motor impairment after stroke: a pilot reliability study. J Neurol Neurosurg Psychiatry 1990;53:576-9.

23. Duncan PW, Lai SM, Bode RK, Perera S, De Rosa J. Stroke Impact Scale-16: a brief assessment of physical function. Neurology 2003;60:291-6.

24. Palomino Aguado B. Contribution of the adaptation and validation of the Stroke Impact Scale version 16 in the stroke rehabilitation [Spanish] [dissertation]. Madrid: Universidad Completense; 2010.

25. American Thoracic Society/European Respiratory Society. ATS/ERS Statement on respiratory muscle testing. Am J Respir Crit Care Med 2002;166:518-624 
26. Calaf N. Assessment of the maximal respiratory pressures [Spanish]. In: Scientific Committee of SEPAR. Manual procedures of SEPAR 4. Assessment evaluation of pulmonary function II. Barcelona: Sociedad Española de Neumología y Cirugía Torácica; 2004. p. 134-44.

27. Fiz JA, Haro M, Aguilar J, et al. Spirometry and maximal respiratory pressures in patients with facial paralysis. Chest 1993;103:170-3.

28. Morales P, Sanchis J, Cordero PJ, Díez JL. Maximum static respiratory pressures in adults. Reference values for a Caucasian Mediterranean population [Spanish]. Arch Bronconeumol 1997;33:213-9.

29. Dancey C, Reidy J. Statistics without maths for psychology: using SPSS for Windows. London: Prentice Hall; 2004.

30. Hautmann H, Hefele S, Schotten K, Huber RM. Maximal inspiratory mouth pressures (PIMAX) in healthy subjects-what is the lower limit of normal? Respir Med 2000;94:689-93.

31. Polese JC, Pinheiro MB, Faria CD, Britto RR, Parreira VF, Teixeira-Salmela LF. Strength of the respiratory and lower limb muscles and functional capacity in chronic stroke survivors with different physical activity levels. Braz J Phys Ther 2013;17:487-93.

32. Terzi N, Corne F, Mouadil A, Lofaso F, Normand H. Mouth and nasal inspiratory pressure: learning effect and reproducibility in healthy adults. Respiration 2010;80:379-86.

33. Wen AS, Woo MS, Keens TG. How many maneuvers are required to measure maximal inspiratory pressure accurately. Chest 1997;111:802-7.

34. Fugl-Meyer AR, Linderholm H, Wilson AF. Restrictive ventilator dysfunction in stroke: its relation to locomotor function. Scand J Rehabil Med Suppl 1983;9:118-24.

35. Enright PL, Kronmal RA, Manolio TA, Schenker MB, Hyatt RE. Cardiovascular Health Study Research Group. Respiratory muscle strength in the elderly: correlates and reference values. Am J Respir Crit Care Med 1994;149:430-8.

36. Costa R, Almeida N, Ribeiro F. Body position influences the maximum inspiratory and expiratory mouth pressures of young healthy subjects. Physiotherapy 2014;101:239-41.

37. Annoni JM, Ackermann D, Kesselring J. Respiratory function in chronic hemiplegia. Int Disabil Stud 1990;12:78-80.

38. Odia GI. Spirometry in convalescent hemiplegic patients. Arch Phys Med Rehabil 1978;59:319-21.

39. Perry J, Garrett M, Gronley JK, Mulroy SJ. Classification of walking handicap in the stroke population. Stroke 1995;26:982-9.

40. Kim K, Fell DW, Lee JH. Feedback respiratory training to enhance chest expansion and pulmonary function in chronic stroke: a double-blind, randomized controlled study. J Phys Ther Sci 2011;23:75-9.

41. Boitano L. Management of airway clearance in neuromuscular disease. Respir Care 2006;51:913-22. discussion 922-2.

42. McCool FD. Global physiology and pathophysiology of cough: ACCP evidence-based clinical practice guidelines. Chest 2006;129(1 Suppl): 48S-53S.

43. Hannawi Y, Hannawi B, Rao CP, Suarez J, Bershad E. Stroke-associated pneumonia: major advances and obstacles. Cerebrovasc Dis 2013;35:430-43.

44. Martino R, Foley N, Bhogal S, Diamant N, Speechley M, Teasell R. Dysphagia after stroke: incidence, diagnosis, and pulmonary complications. Stroke 2005;36:2756-63.

45. Sellars C, Bowie L, Bagg J, et al. Risk factors for chest infection in acute stroke: a prospective cohort study. Stroke 2007;38:2284-91.

46. Kulnik S, Rafferty G, Birring S, Moxham J, Kalra L. A pilot study of respiratory muscle training to improve cough effectiveness and reduce the incidence of pneumonia in acute stroke: study protocol for a randomized controlled trial. Trials 2014;15:123.

47. Kulnik S, Birring S, Moxham J, Rafferty G, Kalra L. Does respiratory muscle training improve cough flow in acute stroke? Pilot randomized controlled trial. Stroke 2015;46:447-53. 